\title{
Developmental trajectories of body mass index throughout the life course: an application of Latent Class Growth (Mixture) Modelling
}

\section{Trynke Hoekstra}

VU University, Faculty of Earth and Life Sciences, Department of Health Sciences and the EMGO Institute for Health and Care Research, Amsterdam, Netherlands

VU University Medical Centre, Department of Epidemiology and Biostatistics and the EMGO Institute for Health and Care Research, Amsterdam, Netherlands

trynke.hoekstra@vu.nl

\section{Celestina Barbosa-Leiker}

Washington State University, College of Nursing, Spokane, WA

Washington State University, Health and Wellness Services, Pullman, WA

\section{Lando L.J. Koppes}

TNO Quality of Life, Division Work and Employment, Hoofddorp, Netherlands

\section{Jos W.R. Twisk}

VU University, Faculty of Earth and Life Sciences, Department of Health Sciences and the EMGO Institute for Health and Care Research, Amsterdam, Netherlands

VU University Medical Centre, Department of Epidemiology and Biostatistics and the EMGO Institute for Health and Care Research, Amsterdam, Netherlands

(Received July 2010 Revised May 2011)

\section{Abstract}

The aims of this study are 1) to analyse developmental trajectories of body fatness from adolescence into adulthood, thereby determining the number and characteristics of distinct body fatness trajectories, and 2) to relate these distinct subgroups to indicators of cardiovascular disease risk, revealing subgroups specifically at risk. This paper will illustrate in more detail the application of Latent Class Growth (Mixture) Modelling (LCGMM) on longitudinal, observational data. Data were obtained from the Amsterdam Growth and Health Longitudinal Study, an ongoing observational study of apparently healthy participants ( $n=336)$. Participants were followed up from 13-42 years of age. Body Mass Index was used as a marker for body fatness and cardiovascular diseases (CVD)-risk factors included Mean Arterial Pressure and HDL-Cholesterol. LCGMM was used for the identification of developmental trajectories of body fatness, and linear regression analyses were used for the associations between the trajectories and CVD-risk. Analyses revealed three distinct trajectories; a "normative" trajectory (88.4\%), a progressively overweight trajectory (4.5\%) and a progressively overweight but stabilising trajectory (7.1\%). Significant differences in CVD-risk between these trajectories appeared. These results show that body fatness development throughout life is heterogeneous, showing differences in CVD-risk. This paper also demonstrates that LCGMM is a promising technique to distinguish between subjects with different developmental trajectories.

\section{Keywords}

Life course epidemiology, Latent Class Growth (Mixture) Modelling, body fatness, cardiovascular disease risk 


\section{Introduction}

Studying developmental patterns throughout the life course has gained interest over the recent years in the fields of epidemiology, psychology and beyond (Kuh and Ben-Shlomo 2004). More often, longitudinal studies take a life course approach when collecting and analysing data, which offers, through its interdisciplinary framework, (new) opportunities to understand the natural history of chronic disorders such as diabetes, depression or low back pain (Dunn 2010). Especially when studying disease aetiology, a life course approach offers valuable insights in the natural course of a risk factor and subsequent consequences for the chronic disease (Kuh and BenShlomo 2004; Dunn 2010). Various statistical techniques are available when taking a life course approach (Singer and Willett 2003; Kuh and BenShlomo 2004). Most techniques take a so-called variable-centred approach (Muthén and Muthén 2000) which focuses on relationships among variables in principle; the goal is to predict outcomes or to study the influence of certain risk factors on disease outcomes. Examples are regression models and structural equation models. Often, a different approach, namely a person-centred approach can offer more insight than a variable-centred approach when wanting to account for population heterogeneity within the sample. Person-centred techniques focus on relationships among individuals, aiming to group similar individuals based on their characteristics (Muthén and Muthén 2000). Latent Class Models, specifically Latent Class Growth (Mixture) Modelling (LCGMM) are good examples of such a technique (Jung and Wickrama 2008; Muthén and Muthén 2000; Muthén 2006; Muthén 2002; Muthén 2004; Muthén and Muthén 2010; Nagin 1999). This paper will apply these techniques, following a rise in interest within life course research (Dunn 2010). As an example, we will use data from a relevant field of research; we will study the course of body fatness in relation to cardiovascular diseases (CVD). As it is well known that the prevalence of CVD is increasing rapidly across all age groups over the world (World Health Organisation 2007), a range of approaches has been undertaken to prevent the onset of CVD. Examples of such attempts are mainly weight loss and weight management programmes
(Groeneveld et al 2008), but few have shown effectiveness. The application of LCGMM can give more insight in the heterogeneity of the (natural) development of obesity throughout the life course. Assessing cardiovascular disease consequences of distinct developmental patterns can provide opportunities for more targeted prevention of cardiovascular diseases, to increase (long-term) effectiveness. Moreover, the limited papers available utilising these techniques highlight the need for more papers, as it is becoming increasingly clear that in many fields of research (amongst others, in the field of cardiovascular diseases), multiple pathways to developing (chronic) diseases exist (Patrick and Schulenberg 2011; Schulenberg et al 2005; Dunn et al 2006; van Leeuwen et al 2011; Joinson et al 2009; Zavrelova et al 2011) therefore justifying taking into account this heterogeneity when analysing such data.

The aim of the current study is threefold. Our first aim is to analyse developmental patterns of body fatness from adolescence into adulthood, thereby determining the number and characteristics of distinct body fatness trajectories. Secondly, we aim to relate these distinct subgroups to indicators of cardiovascular disease risk (namely high-density lipoprotein (HDL)-cholesterol and mean arterial pressure, MAP) later in life, revealing subgroups specifically at risk. Thirdly, and overall, this paper will illustrate in more detail the application of LCGMM to longitudinal, observational data.

\section{Materials and methods}

\section{Study design}

The Amsterdam Growth and Health Longitudinal Study (AGAHLS) is an observational longitudinal cohort study which started in 1976. Approximately 650 boys and girls (mean age of 13 years) from the first two grades of two secondary schools in the Netherlands were included in the study. The initial goals of the study were to describe the natural development of growth, health and lifestyle and further to investigate associations between health and lifestyle. During the adolescence period, participants were measured annually, and thereafter, six more examinations took place, of which the most recent took place in 2006 (mean age of 42 years). 
Medical ethical approval was obtained from the Medical Ethics Committee of the VU University Medical Centre, and written informed consent was obtained from each participant, as well as from the parent or guardian (during adolescence).

More detailed information on study design and specific measurements at each examination has been published elsewhere (Kemper 1995; Kemper 2004).

\section{Inclusion and exclusion criteria}

For the current study, participants from whom valid measurements of the cardiovascular disease risk factors were available and who had at least one measurement of body fatness over the previous 30 years, were included. If women were pregnant during a particular round of measurement, that measurement (for body fatness) was not included in the analyses. In total, 176 women (52.4\%) and 160 men were included in the study (total $n=336$ ).

\section{Body fatness measurements}

Body fatness was quantified by the Body Mass Index (BMI). BMI was calculated by dividing body mass (kilograms) by body height squared (meters squared).

\section{Indicators of cardiovascular disease risk}

Fasting HDL cholesterol values were determined from blood drawn from the antecubital vein (Roche diagnostics, Mannheim, Germany). Blood pressure was measured every 5 minutes for 60 minutes using an automated device (Dinamap Procare 100). Subsequently, mean arterial pressure (MAP) was calculated as $((2 *$ diastolic blood pressure + systolic blood pressure) / 3) (Sesso et al 2000).

\section{Lifestyle covariates}

Habitual energy intake was measured using a detailed cross-check dietary history face-to-face interview method, based on Beal (Beal 1967) and Marr (Marr 1971), and expressed in kilojoules per day. The interview was adapted for the AGAHLS. For the analyses, mean life course food intake was calculated in kilojoules per day.

Physical activity in minutes per week was measured using a detailed questionnaire, especially designed and validated for the AGAHLS (Verschuur
1987). For the analyses, mean life course physical activity was calculated in minutes per week.

Smoking behaviour was measured with a separate questionnaire (Bernaards et al 2004), from which participants were categorised in one of three categories; never smoked, ever smoked (but not a current smoker), or current smoker.

Alcohol consumption was also measured with a short questionnaire (Koppes et al 2002), determining whether participants were alcohol drinkers or not (at the last time point).

\section{Statistical analyses}

Statistical analyses were conducted using the Mplus 6.1 ( Muthén and Muthén 2011; Muthén and Muthén 2010) and the SPSS 17.0 software packages. The analyses for the current study consisted of two steps, described in detail below.

\section{Step 1. (Piecewise) Latent Class Growth (Mixture) Modelling}

Heterogeneity in longitudinal development of body fatness was analysed with (Piecewise (defined in Procedures below)) Latent Class Growth (Mixture) Modelling (LCGMM) (Muthén and Muthén 2010; Muthén and Muthén 2000; Muthén 2006; Muthén 2002). LCGMM is a contemporary longitudinal technique based on structural equation modelling, incorporating both continuous and categorical latent (unobserved) variables. The technique is an extension of conventional growth modelling. In conventional analyses, the assumption that all individuals in the study sample come from a single population should hold; meaning that one (average) trajectory will adequately describe the developmental pattern of the sample. This assumption is relaxed in LCGMM, meaning that individuals in the sample need not come from one single underlying population, but can come from multiple, underlying (or latent) subpopulations. Identifying the number and characteristics of these underlying sub-populations is the main aim of LCGMM. This is done by identifying $k$ number of distinct latent classes (i.e. subgroups) of, in the present study, trajectories of body fatness. Each identified class has its own specific growth parameters (intercept, slope), which are also assumed to be unobserved, or latent. Furthermore, 
LCGMM can also account for within-class variation in either, or both, of the growth factors, implying within-class heterogeneity on top of the betweenclass heterogeneity in initial status (intercept) or growth (slope).

\section{Procedures}

Various LCGMM models were run before choosing a final model. First, several linear LCGMM with fixed intercept and slope variance within-classes were investigated. Next, quadratic slopes were added to the model, allowing for curved developmental patterns. Thirdly, we investigated piecewise models which allowed for different phases in development. Models with three pieces were investigated, showing possibilities of different growth rates (and directions) during each phase. Phase one was defined as the adolescence phase (age 13-16), phase two was defined as the young adulthood phase (age 21-29) and phase three was defined as the adulthood phase (age 32-42).

For all models, a one to four class solution was modelled, starting with a one class solution (i.e. there are no subgroups within the data; all individuals follow the same trajectory over time), then adding classes one at a time.

After the choice for a final model was made, necessity for the estimation of within-class intercept and slope variance was assessed. The assessment was made based on another stepwise procedure; step by step, intercept and/or slope variances within classes were estimated in the model. After each step, model fit and clinical interpretation was assessed. Caution was taken when dealing with estimated slope variance, according to the current literature (Muthén and Muthén 2000; Muthén and Muthén 2010; Nylund et al 2007; Jung and Wickrama 2008).

\section{Model selection (class identification)}

To determine the optimal number of latent classes, we used two model fit indices; the Bayesian Information Criterion (BIC), and the bootstrap Likelihood Ratio Test (BLRT). The BIC (Schwarz 1978) is commonly used within mixture modelling analyses, considering both the likelihood of the model as well as the number of parameters in the model; a lower BIC value indicates a better model fit (a decrease of at least 10 points shows a sufficient improvement) (Raftery 1995). The BLRT uses bootstrap samples to estimate the distribution of the log likelihood difference test statistic. The test provides a $p$-value, indicating that a model with one class less ( $k-1$ class model) has to be rejected in favour of the $k$ class model. The BLRT has been shown to be a very consistent indicator of the optimal number of classes (Nylund et al 2006). To further determine the optimal number of classes, we looked at the posterior probabilities for each individual in the sample (Nylund et al 2006). For each individual in the sample, the probability of belonging to each of the $k$ classes is estimated. The probability for the class to which the individual was assigned, should be considerably higher than the other probability/probabilities. By this means, the classes are clearly distinguished from each other. Finally we considered the usefulness of the separate classes in practice.

\section{Technical issues}

Missing data were handled according to the Expectation-Maximation Algorithm (EM-Algorithm) (Dempster et al 1977; Muthén and Shedden 1999). An important assumption of this algorithm is that the data should be missing at random (MAR). Although this assumption is difficult to test (Pothoff et al 2006), the included participants were compared with the excluded participants on the variables analysed. Regarding BMI at baseline (i.e. in 1976 at age 13), these two groups did not differ; the average BMI of the included participants was 17.39 (1.88), compared to 17.77 (2.23) for the excluded participants. Further, of the included participants, $52.4 \%$ were female, compared with $52.8 \%$ of the excluded participants. In the Mplus program (Muthén and Muthén 2010), it is in addition possible to extract missing data patterns to study the missing data in further detail. These assessments did not point to selective missing data either, corresponding to earlier research with data from the Amsterdam Growth and Health Longitudinal Study (see for example te Velde et al 2004).

LCGMM models are computationally-heavy models, often with convergence issues or hitting local maxima. Mplus incorporates random starts to avoid these issues as much as possible (Muthén and Muthén 2011; Muthén and Muthén 2010; Jung and Wickrama 2008). In the current paper, 750 random starting values were applied with 50 final optimisations. Only solutions with 
replicated log likelihoods were accepted, where the OPTSEED procedure can help in making a final decision (Jung and Wickrama 2008; Muthén and Muthén 2010). Because LCGMM are complicated models, often problems arise when estimating more than three classes (Jung and Wickrama 2008; Muthén and Muthén 2010). By taking into account this complexity, together with the sample size and the number of hypothesized trajectories from previous studies (Mustillo et al 2003; Li et al 2007), we modelled a maximum of four trajectories.

\section{Step 2. Linear Regression}

The first step of the analyses provided us with a classification of the study sample into distinct trajectory subgroups, coded as a categorical variable with $k$ number of categories. To study the association between the distinct trajectories and CVD risk (i.e. mean arterial pressure and HDL values) later in life (i.e. at 42 years of age), several linear regression analyses were performed. First, crude analyses were performed, in which class membership was related to the CVD risk indicators, correcting for gender. Second, adjusted analyses were carried out, adding the lifestyle covariates, described above, to the regression model.

\section{Results}

Illustrating the study sample, table 1 shows descriptive data for all subjects for body fatness (BMI) at each time point. Mean values seem to increase throughout the life course. Mean (standard deviation) HDL levels at age 42 for the total study population were 1.725 (0.423) mmol/l. Mean (standard deviation) MAP at age 42 was 84.970 (9.889). $57.9 \%$ of the study sample reported having never smoked up to age 42 , whereas $26.6 \%$ did smoke in the past, but were not current smokers. Only $8.8 \%$ had never drunk alcohol at age 42 .

\section{Table 1. Descriptive information (mean (standard deviation)) regarding the development of body fatness}

$\begin{array}{lll}\text { Age } & \text { Sample (Females, Males) } & \begin{array}{l}\text { BMI }\left(\mathbf{k g} / \mathbf{m}^{2}\right), \text { SD } \\ \text { Females }\end{array} \\ 13 & 168,153 & 17.81(2.14) \\ 14 & 155,124 & 18.66(2.08) \\ 15 & 151,121 & 19.41(2.14) \\ 16 & 153,122 & 20.01(2.10) \\ 21 & 74,57 & 21.65(2.48) \\ 27 & 73,60 & 22.03(2.36) \\ 29 & 64,61 & 22.46(2.58) \\ 32 & 169,145 & 22.85(3.13) \\ 36 & 157,143 & 23.57(3.47) \\ 42 & 175,160 & 24.09(3.87)\end{array}$

\section{(Piecewise) Latent Class Growth (Mixture) Models}

Table 2 shows the results of the (Piecewise) Latent Class Growth (Mixture) Models (LCGMM). First, several linear models (one-four classes) were conducted and compared. These models are shown in the top four rows of the table, pointing towards a three or four class solution. These four solutions were subsequently compared to the next four solutions which permit quadratic development in the class(es). However, the quadratic models with three and four classes are increasingly complex and lead to convergence problems. In our case, we had problems with the estimation of the standard errors due to a

$\begin{array}{ll}\text { BMI }\left(\mathrm{kg} / \mathrm{m}^{2}\right), \text { SD } & \text { BMI }\left(\mathrm{kg} / \mathrm{m}^{2}\right), \text { SD } \\ \text { Males } & \text { Study sample } \\ 16.93(1.42) & 17.39(1.88) \\ 17.68(1.49) & 18.23(1.90) \\ 18.38(1.66) & 18.95(2.01) \\ 19.21(1.70) & 19.66(1.97) \\ 21.20(1.67) & 21.45(2.17) \\ 22.46(2.17) & 22.22(2.28) \\ 22.94(2.22) & 23.70(2.41) \\ 23.79(2.41) & 23.28(2.85) \\ 24.60(2.68) & 24.06(3.16) \\ 25.20(2.92) & 24.62(3.49)\end{array}$

non-positive definite information matrix, and difficulties in estimating the quadratic slopes for some classes that appeared to include two or three individuals only. Therefore, for the quadratic models we had not four, but two models to compare. Next, four models with piecewise trajectories (piece one was defined by the ages 13-16, piece two by the ages 21-29 and piece three was defined by the ages 32-42) were conducted. It can be seen that the improvement of these models (specifically indicated by much lower $\mathrm{BIC}$ values) is clear. 


\section{Table 2. (Piecewise) Latent Class Growth (Mixture) Models Results}

\section{Number of classes}

1, linear slope

2 , linear slope

3, linear slope

4, linear slope

1, quadratic slope

2 , quadratic slope

1, Piecewise, linear slope

2 , Piecewise, linear slope

3, Piecewise, linear slope ${ }^{2}$

4, Piecewise, linear slope ${ }^{2}$

class 1: 19.498

class 2: 25.892

class $3: 16.997$

class 4: 20.238
Slopes $^{1}$

0.269

0.261

0.434

0.258

0.140

0.479

0.465

0.259

0.267

0.119

$0.436 /-0.007$

$0.530 /-0.003$

$0.431 /-0.007$

$0.743 / 1.208 / 0.538$

$0.752 / 1.159 / 0.497$

$0.515 / 2.299 / 1.528$

$0.796 / 1.130 / 0.566$

$0.551 / 1.514 /-0.322$

$0.517 / 2.313 / 1.502$

$0.681 / 2.410 / 1.294$

$0.184 / 0.824 / 2.080$

$0.771 / 1.116 / 0.555$

$0.525 / 1.431 /-0.302$
BIC

BLRT

9899.16

9884.609

9887.077

9894.435

8671.655

8639.308

8283.726

8262.715

8265.720

8274.552

Not calculated

$\mathrm{P}<0.001$

$P<0.001$

$P=0.034$

Not calculated

$\mathrm{P}<0.001$

$P<0.001$
Prob. Subjects per class

$1.000 \quad 336$

$0.95614 / 322$

Not calculated $\quad 1.000 \quad 336$

$\mathrm{P}<0.001 \quad 0.936 \quad 324 / 12$

$0.867 \quad 297 / 24 / 15$

$0.91016 / 2 / 294 / 24$

Notes.

1. All values indicate linear slopes, except for the 1-and 2-class quadratic models where the second value indicates a quadratic slope

2. Piece one is defined by the ages 13-16, piece two is defined by the ages $21-29$ and piece three is defined by the ages 32-42 


\section{Choosing the final model}

Comparing the three sets of four models based in model fit alone was complicated, as often the different model fit indices are not in agreement with each other (Nylund et al 2007). Based on the BIC, for example, the "best" model (i.e. the model with the lowest value) is the two class piecewise model, although the BIC of the three class piecewise model is almost the same (a difference of three points). Literature advises about "significant" improvement in the BIC-values; improvement of at least 10 points indicates a sufficient improvement of the model (Raftery 1995), indicating that based on the BIC, the two and three class piecewise models have equivalent fit. Further, based on the BLRT, the "best" model is a four class model (indicated by a nonsignificant $p$-value). Both (BIC and BLRT) model fit indices therefore do not point consistently to one definite solution (Nylund et al 2007). Hence, we also took the clinical interpretation of the trajectories into account. Based on this, our final model was a three class piecewise model, showing a "normative" BMI trajectory ( $n=297)$, a progressively overweight, but stabilising trajectory $(n=24)$ and a progressively overweight $(n=15)$ trajectory.

When the choice for the number of classes in the final model has been made, the necessity of random intercept or slope variance within class was assessed. The results of these further analyses are not shown in the table, as none of the additional models showed a sufficient increase in model indices. Based on existing literature (Jung and Wickrama 2008), the ultimate final model was the most parsimonious model; a three class piecewise model without random intercept and slope variance within classes (shown in bold in Table 2). Figure 1 shows mean trajectories of the final model and Table 3 provides descriptive information of the three trajectories regarding the confounders used in the regression models.

Figure 1. Estimated trajectories of Body Mass Index (Y-axis) from the age of 13 to 42 years (X-axis)

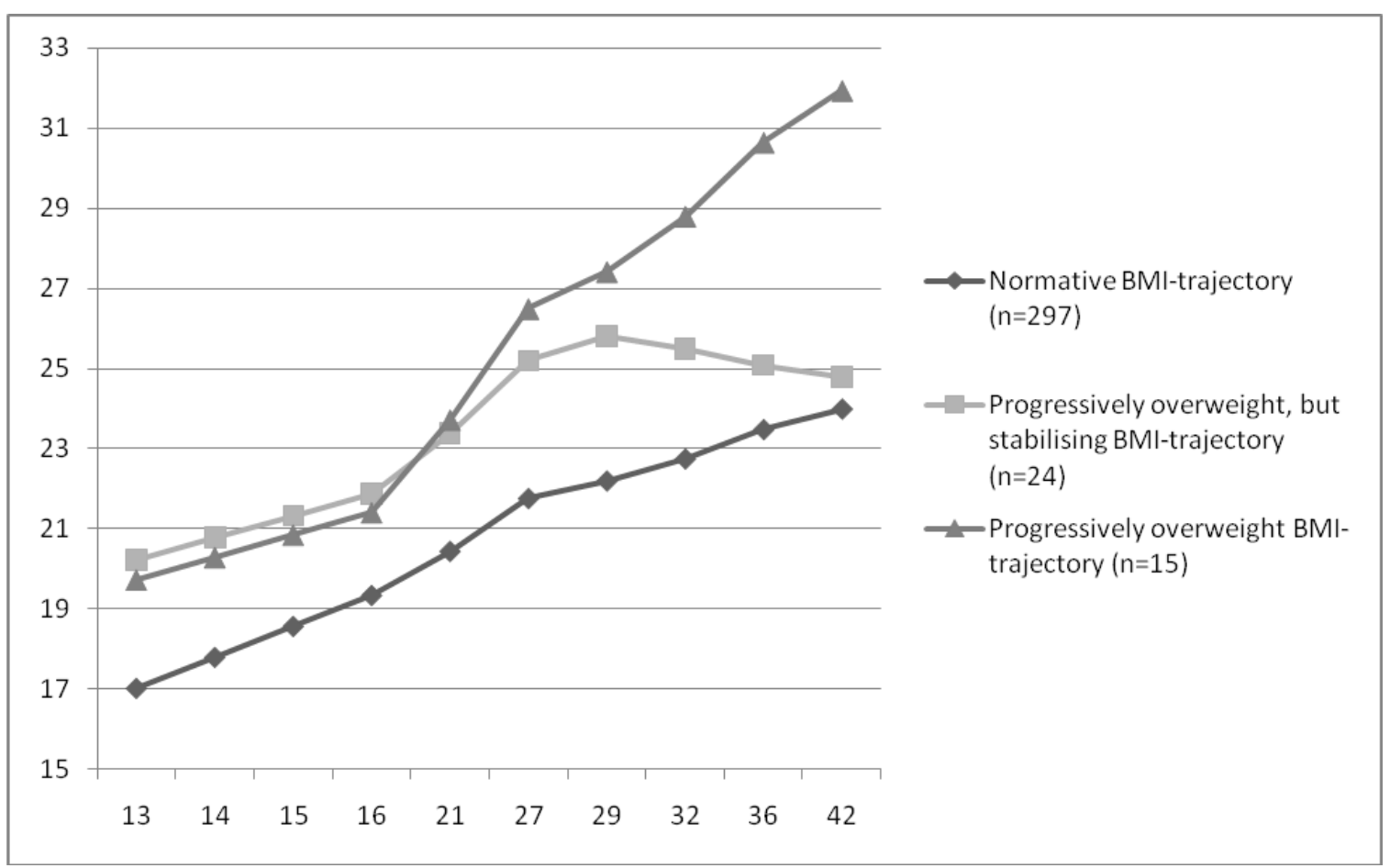


Table 3. Descriptive information of the three estimated trajectories of Body Mass Index

$\begin{array}{llll}\text { Variables of interest } & \begin{array}{l}\text { Normative } \\ \text { trajectory }\end{array} & \begin{array}{l}\text { Overweight, but } \\ \text { stabilising trajectory }\end{array} & \begin{array}{l}\text { Progressively overweight } \\ \text { trajectory }\end{array} \\ \begin{array}{l}\text { Gender } \\ \text { \% female }\end{array} & 49.2 & 79.2 & 73.3 \\ \begin{array}{l}\text { Smoking } \\ \% \text { smoker at age } 42\end{array} & 15.9 & 4.2 & 26.7 \\ \begin{array}{l}\text { \% previous smoker } \\ \text { Alcohol intake }\end{array} & 26.7 & 25.0 & 26.7 \\ \begin{array}{l}\text { \% drinkers } \\ \text { Total Physical Activity } \\ \text { (min/week) }\end{array} & 92.2 & 95.8 & 66.7 \\ \begin{array}{l}\text { Average (SD) } \\ \text { Nutritional intake }\end{array} & 559.33(179.52) & 472.28(159.49) & 424.73(62.96) \\ \begin{array}{l}\text { (KJ/day) } \\ \text { Average (SD) }\end{array} & & & \\ \end{array}$

\section{Linear Regressions}

Table 4 summarises the results of the linear regression analyses investigating the associations between trajectory membership and MAP and HDL values at the age of 42 years.

For MAP, both the overweight, but stabilising and the progressively overweight classes were significantly different from the normative class. For
HDL, the progressively overweight class showed significantly lower values compared to the two other classes. From the two adjusted models, it becomes clear that for both the analyses with MAP as well as $\mathrm{HDL}$, gender is the most important confounder. After adding the lifestyle covariates, the effects in both models are much less affected.

Table 4. Linear Regression Analyses showing associations between class membership and indicators of CVD-risk

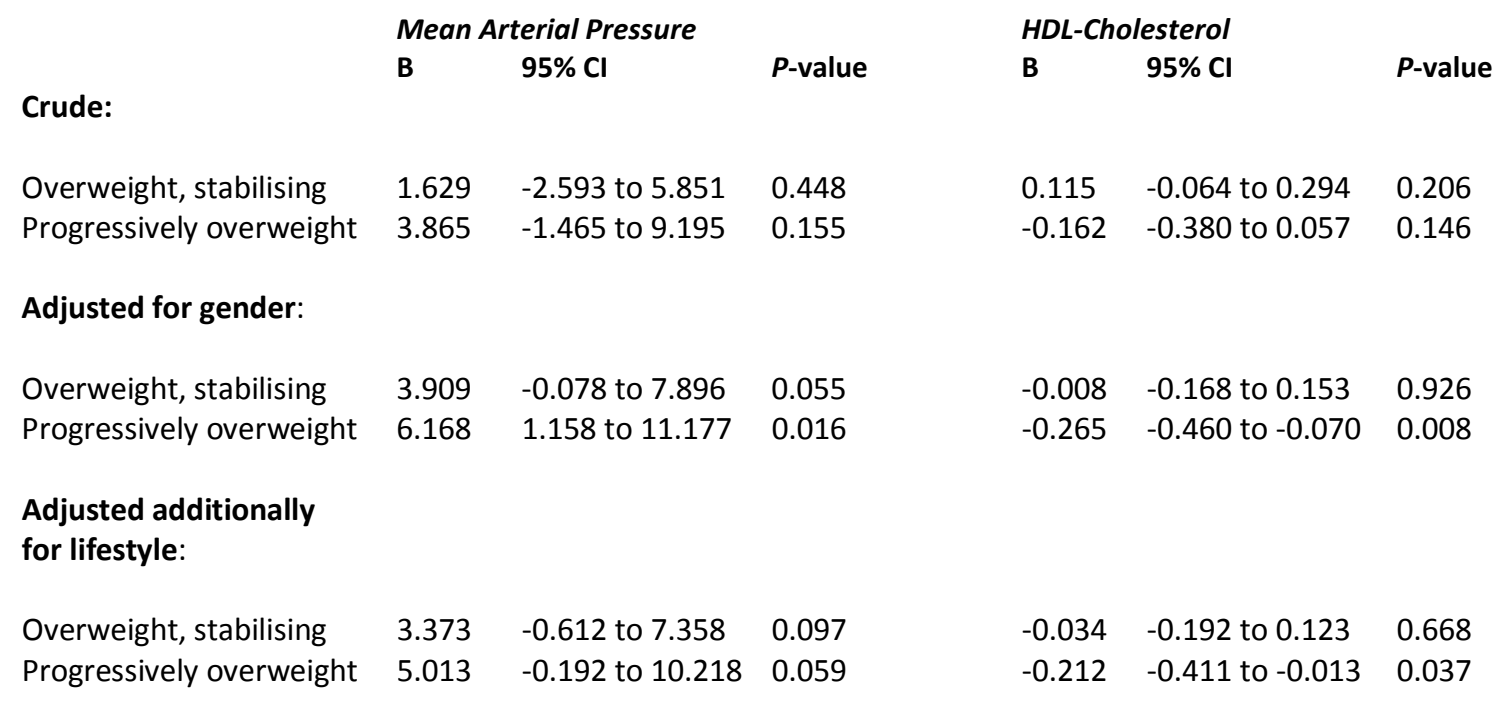

The "normative" trajectory was set as reference 


\section{Discussion}

The current study gave particular attention to the application of Latent Class Growth (Mixture) Models on life course data. As an example, we investigated the possible existence of distinct life course trajectories of body fatness, in an apparently healthy sample. Developmental patterns of body mass index were studied from the age of 13 to 42 years, where three distinct trajectories emerged. Subsequently, class membership was related to CVD risk factors at the age of 42 years.

\section{Researching heterogeneity in development of body fatness}

The acknowledgement of the existence of heterogeneity within a study sample has gained interest over the past years. Especially within the field of alcohol research, recognising heterogeneity has led to theories of multiple developmental pathways of alcohol abuse (Schulenberg et al 1996; Maggs and Schulenberg 2002; Brennan et al 2011). Slowly, similar approaches are emerging in other fields of research (van Leeuwen et al 2011; Mustillo et al 2003; Dunn et al 2006). Comparing the analysis of heterogeneity of body fatness development with existing studies, shows that limited research has been conducted and methodologies differ a great deal. Studies with methods comparable to ours are, for example, the study by Li et al (2007). They studied developmental trajectories of BMI focussing only on childhood (American children of age 2 to 12 years). The authors found a three class solution to be the best option, containing a normative group, a consistently overweight group and a late-onset overweight group. Another study, by Mustillo et al (2003), also studied developmental patterns of body fatness, but with a slightly different statistical technique implemented in the SAS software (Nagin 1999). They studied American children from the age of 9 to 16 years, and found four distinct trajectories, where most children were classified in the group with a very low prevalence of obesity. A third study (Ostbye et al 2011), following up adults from the age of 18-49, also conducted the analyses in the SAS program, and analysing continuous BMI-values in their models is worth mentioning in the light of our study. Their sample, more heterogeneous in terms of race and socioeconomic status and larger compared to our sample, revealed four trajectories, three of them comparable to our trajectories. The papers highlighted above show consistently that body fatness development is heterogeneous throughout the life course, in various study populations. This indicates that researchers should be aware of this when analysing their data.

There are other noteworthy studies, which investigate differences in development of body fatness retrospectively, seeing differences in the development of body fatness between individuals at high and low risk of coronary artery disease (Youssef et al 2002), between people with and without the metabolic syndrome (Ferreira et al 2005) and also between people with high and low adult body fat (Nooyens et al 2007). Although the aim of these studies was also to investigate developmental patterns of risk factors, they were primarily interested in differencein development between patients and non-patients. This is an a priori categorisation of the study participants into predefined groups on the basis of their disease status, whereas the categorisation with LCGMM is on the basis of the developmental trajectory of the risk factor per se. When choosing a statistical method incorporating a heterogeneity component, one should keep in mind the differences between the techniques available, to allow for answering the research questions appropriately.

\section{Methodological issues}

In the current literature, there is discussion about the usage of Latent Class Growth (Mixture) Models within epidemiology. Models are mathematically complicated and care should be taken when applying them, as a wide range of possibilities is available for the specification of the model. The debate is mainly between the choice between a Latent Class Growth Model (LCGM) and a Latent Class Growth Mixture Model (LCGMM). In short, the LCGM methodology was developed by Nagin and colleagues (Roeder et al 1999; Nagin and Tremblay 2001a; Nagin 1999; Nagin and Tremblay 2001b; Jones et al 2001) and is implemented in the SAS software, whereas the LCGMM methodology was developed by Muthén and colleagues and implemented in the Mplus software 
(LCGM is also possible in this framework) (Muthén and Muthén 2011; Muthén and Muthén 2000; Muthén and Muthén 2010; Muthén 2006; Muthén 2002). The difference between the two methods is the assumptions both have about the underlying (latent) populations. LCGM assumes no within-class variation (the variance within each class is fixed to zero), whereas LCGMM relaxes this assumption by allowing the within-class variance to be estimated. Hereby, LCGMM allows for additional heterogeneity in the model, thus being the more flexible technique. Because of this flexibility however, models can have difficulty arriving at a proper statistical solution. Although the underlying aims of both techniques are similar (namely, identifying distinct developmental trajectories), the approach can vary. It is important for researchers to clearly describe which approach they used, to allow for objective comparison and interpretation of the results.

Another important point of discussion is the decision of the number of classes (or subgroups). Several model fit indices are available to aid the researcher with this decision. However, these fit indices are not always consistent (Nylund et al 2007). Although an interesting simulation study has been published showing that the BLRT is the most consistent fit index, controversy remains. Therefore, in this paper, several indices are reported and all are used in deciding on the final model.

\section{General remarks}

We have shown that LCGMM is a promising technique to distinguish between subjects with different developmental trajectories. It can help researchers in the field of obesity and cardiovascular disease risk specifically and epidemiology in general, amongst others, to gain insight in the relationships between developmental patterns of risk factors for chronic disease and provide interesting novel research opportunities. However, researchers should use caution when utilising these complex techniques, as they still have to cope with technical issues that have not all been resolved. Careful application, with careful interpretation of the results, can, however, bring valuable insights in many fields of research and instigate new research ideas.

We have shown that elaborate comprehension of heterogeneity in the progress of risk factors for (preventable) chronic diseases is interesting for aetiology and prevention. Our results have shown that body fatness development from adolescence into adulthood is heterogeneous, consistent with previous studies (Li et al 2007; Mustillo et al 2003; Ostbye et al 2011). Trajectory membership was associated with CVD risk at age 42 even if lifestyle variables are taken into account. This indicates that fatness trajectory shape matters for the magnitude of CVD risk in this study population. However, effects were small and our study sample is relatively healthy, making it difficult to generalise these findings to other study samples or the general population. The AGAHLS is one of few studies following up participants for over thirty years, throughout life, making these findings very valuable. Further research, studying the technical issues LCGMM are currently experiencing, should be conducted, but also continued application of these models on data from diverse fields of research is warranted, to continue to explore the usability and applicability of these models.

\section{References}

Beal VA. (1967) The nutritional history in longitudinal research. Journal of the American Dietetic Association, 51, 426-432.

Bernaards CM, Twisk JWR, Mechelen W van, Snel J and Kemper HCG. (2004) Comparison between self-report and a dipstick method (NicCheck 1) to assess nicotine intake. European Addiction Research, 10,163-167.

Brennan P, Schutte K, Moos B and Moos R. (2011) Twenty-year alcohol-consumption and drinking-problem trajectories of older men and women. Journal of Studies of Alcohol, 72, 308-321.

Dempster AP, Laird NM and Rubin DB. (1977) Maximum likelihood from incomplete data via the EM algorithm. Journal of the Royal Statistical Society, Series B, 39, 1-39.

Dunn KM. (2010) Extending conceptual frameworks: life course epidemiology for the study of back pain. BMC Musculoskeletal Disorders, 2, 11-23. 
Dunn KM, Jordan K and Croft PR. (2006) Characterizing the course of low back pain: a latent class analysis. American Journal of Epidemiology, 163, 754-761.

Ferreira I, Twisk JWR, Mechelen W van, Kemper HCG and Stehouwer CDA. (2005) Development of fatness, fitness, and lifestyle from adolescence to the age of 36 years: determinants of the metabolic syndrome in young adults: the Amsterdam Growth and Health Longitudinal Study. Archives of Internal Medicine, $165,42-48$.

Groeneveld IF, Proper KI, Beek AJ van der, Duivenbooden C van and Mechelen W van. (2008) Design of a RCT evaluating the (cost-) effectiveness of a lifestyle intervention for male construction workers at risk for cardiovascular disease: The Health under Construction study. BMC Public Health, 8. I.

Joinson C, Heron J, Butler R and Croudace TJ. (2009) Development of nighttime bladder control from $4-9$ years: association with dimensions of parent rated child maturational level, child temperament and maternal psychopathology. Longitudinal and Life Course Studies, 1, 73-94.

Jones BL, Nagin DS and Roeder K. (2001) A SAS procedure based on mixture models for estimating developmental trajectories. Sociological Methods and Research, 29, 374-393.

Jung T and Wickrama KAS. (2008) An introduction to Latent Class Growth Analysis and Growth Mixture Modeling. Social and Personality Psychology Compass, 2, 302-317.

Kemper HCG. (2004) Amsterdam Growth and Health Longitudinal Study: A 23-year follow-up from teenager to adult about lifestyle and health. In J Borms, M Hebbelinck and AP Hills, eds. Medicine and Sport Science. Pp. 1-20, Karger, Basel, Switzerland.

Kemper, HCG. (1995) The Amsterdam Growth Study: A longitudinal analysis of health, fitness and lifestyle. Human Kinetics, Champaign, IL, USA.

Koppes LL, Twisk JWR, Snel J and Kemper HCG. (2002) Concurrent validity of alcohol consumption measurement in a "healthy" population; quantity-frequency questionnaire versus dietary history interview. British Journal of Nutrition, 88, 427-434.

Kuh D and Ben-Shlomo Y. (2004) A life course approach to chronic disease epidemiology. Oxford University Press, Oxford.

Leeuwen C van, Post M, Hoekstra T, Der Woude L van, Groot S de, Snoek G, Mulder D and Lindeman E. (2011) Trajectories in the course of life satisfaction after spinal cord injury: identification and predictors. Archives of Physical Medicine and Rehabilitation, 92, 207-213.

Li C, Goran MI, Kaur H, Nollen N and Ahluwalia JS. (2007) Developmental trajectories of overweight during childhood: role of early life factors. Obesity, 15, 760-771.

Maggs J and Schulenberg J. (2002) Trajectories of alcohol use during the transition to adulthood. Alcohol Research and Health, 28, 195-201.

Marr JW. (1971) Individual dietary surveys: purpose and methods. World Review of Nutrition and Dietetics, 13, 105-164.

Mustillo S, Worthman C, Erkanli A, Keeler G, Angold A and Costello EJ. (2003) Obesity and psychiatric disorder: developmental trajectories. Pediatrics, 111, 851-859.

Muthén B. (2002) Beyond SEM: General Latent Variable Modeling. Behaviormetrika, 29, 81-117.

Muthén B. (2004) Latent variable analysis: growth mixture modeling and related techniques for longitudinal data. In D. Kaplan ed. Handbook of quantitative methodology for the social sciences. Pp. 345-368, Sage Publications, Newbury Park, CA.

Muthén B. (2006) The potential of growth mixture modelling. Infant and Child Development, 15, 623-625.

Muthén B and Muthén LK. (2000) Integrating person-centered and variable centered analyses: Growth Mixture Modeling with latent trajectory classes. Alcoholism: Clinical and Experimental Research, 24, 882-891.

Muthén B and Muthén LK. (2011) MPlus 6.1 (Computer Program).

Muthén B and Shedden K. (1999) Finite mixture modeling with mixture outcomes using the EM algorithm. Biometrics, 55, 463-469.

Muthén LK and Muthén BO. (2010) Mplus user's guide. Fifth Edition. Muthén and Muthén, Los Angeles, CA.

Nagin DS. (1999) Analyzing developmental trajectories. A semi-parametric group based approach. Psychological Methods, 6, 18-34.

Nagin DS and Tremblay RE. (2001a) Analyzing developmental trajectories of distinct but related behaviors: A group-based method. Psychological Methods, 229, 374-393.

Nagin DS and Tremblay RE. (2001b) Developmental trajectory groups: Fact or a useful statistical fiction? Criminology, 43, 873-904.

Nooyens ACJ, Koppes LL, Visscher TLS, Twisk JWR, Kemper HCG, Schuit AJ, Mechelen W van and Seidell JC. (2007) Adolescent skinfold thickness is a better predictor of high body fatness in adults than is body mass index: the Amsterdam Growth and Health Longitudinal Study. American Journal of Clinical Nutrition, 85, 1533-1539. 
Nylund K, Asparouhov T and Muthén BO. (2007) Deciding on the number of classes in Latent Class Analysis and Growth Mixture Modelling: A Monte Carlo Simulation Study. Structural Equation Modeling, 14, 535-569.

Ostbye T, Malhorta R and Landerman L. (2011) Body mass trajectories through adulthood: results from the National Longitudinal Survey of Youth 1979 Cohort (1981-2006). International Journal of Epidemiology, 40, 240-250.

Pothoff RF, Tudor GE, Pieper KS and Hasselblad V. (2006) Can one assess whether missing data are missing at random in medical studies? Statistical Methods in Medical Research, 15, 213-234.

Patrick M and Schulenberg JE. (2011) How trajectories of reasons for alcohol use relate to trajectories of binge drinking: National panel data spanning late adolescence to early adulthood. Developmental Psychology, 47, 311-317

Raftery A. (1995) Bayesian model selection in social research. Sociological Methodology, 25, 111-163.

Roeder K, Lynch KG and Nagin DS. (1999) Modeling uncertainty in Latent Class Membership: A case study in Criminology. Journal of the American Statistical Association, 94, 766-776.

Schulenberg JE, Merline AC, Johnston LD, O'Malley PM, Bachman JG and Laetz VB. (2005) Trajectories of marijuana use during the transition to adulthood: The big picture based on National Panel data. Journal of Drug Issues, 35, 255-279.

Schulenberg J, O'Malley PM, Bachman JG, Wadsworth K and Johnston L. (1996) Getting drunk and growing up: trajectories of frequent binge drinking during the transition to young adulthood. Journal of Studies of Alcohol, 57, 289-304.

Schwarz GE. (1978) Estimating the dimension of a model. Annals of Statistics, 6, 461-464.

Sesso HD, Stampfer MJ, Rosner B, Hennekens CH, Gaziano M, Manson JE and Glynn RJ. (2000) Systolic and diastolic blood pressure, pulse pressure, and mean arterial pressure as predictors of cardiovascular disease risk in men. Hypertension, 36, 801-807.

Singer J and Willett J. (2003) Applied longitudinal data analysis. Oxford University Press, Oxford.

Velde S te, Ferreira I, Twisk JWR, Stehouwer CD, Mechelen W van and Kemper H. (2004) Birthweight and arterial stiffness and blood pressure in adulthood--results from the Amsterdam Growth and Health Longitudinal Study. International Journal of Epidemiology, 33, 154-161.

Verschuur R. (1987) Daily physical activity and health. Longitudinal changes during the teenage period. PhD thesis (accessed throughout the VU University library; www.ubvu.vu.nl).

World Health Organisation (2007) Cardiovascular diseases - Factsheet number 317.

Youssef AA, Valdez R, Elkasabany A, Srinivasan SR and Berenson GS. (2002) Time-course of adiposity and fasting insulin from childhood to young adulthood in offspring of parents with coronary artery disease: The Bogalusa Heart Study. Annals of Epidemiology, 12, 553-559.

Zavrelova H, Hoekstra T, Alssema M, Welschen L, Nijpels G, Moll AC, Vet H de, Polak B and Dekker J. (2011) Progression and regression: distinct developmental patterns of diabetic retinopathy in patients with type 2 diabetes treated in the diabetes care system West-Friesland, the Netherlands. Diabetes Care, 34, 867-872. 